\title{
EMPIRICAL EVALUATION OF BOUNDARY POLICIES FOR WAVELET-BASED IMAGE CODING
}

\author{
Claudia Schremmer \\ Praktische Informatik IV \\ Universität Mannheim, Germany \\ schremmer@informatik.uni-mannheim.de
}

\begin{abstract}
The wavelet transform has become the most interesting new algorithm for still image compression. Yet there are many parameters within a wavelet analysis and synthesis which govern the quality of a decoded image. In this paper, we discuss different image boundary policies and their implications for the decoded image. A pool of gray-scale images has been wavelet-transformed with different settings of the wavelet filter bank and quantization threshold and with three possible boundary policies.

Our empirical evaluation is based on three benchmarks: a first judgement regards the perceived quality of the decoded image. The compression rate is a second crucial factor. Finally, the best parameter settings with regard to these two factors is weighted with the cost of implementation.

Contrary to the new standard JPEG-2000, where mirror padding is implemented, our investigation proposes circular convolution as the boundary treatment.
\end{abstract}

Keywords: Wavelet Analysis, Image Coding, Boundary Policies, Empirical Evaluation.

\section{INTRODUCTION}

Due to its outstanding performance in compression, new image coding techniques such as the new standard JPEG-2000 [SCE00] [ITU00], focus on the wavelet transform (WT). As we were interested in the influence of the filter length on image coding quality - and contrary to the JPEG2000 standard, where a reversible (Daubechies 5/3-tap) and an irreversible (Daubechies 9/7-tap) wavelet filter bank are proposed - we have investigated the orthogonal and separable wavelet filters developed by Daubechies [Dau92] which belong to the group of wavelets used most often in image coding applications. They specify a number $n_{0}$ of vanishing moments: if a wavelet has $n_{0}$ vanishing moments, then the approximation order of the wavelet transform is also $n_{0}$.

However, implementations of the WT on still images entail other aspects as well: speed, decomposition depth, and boundary treatment policies. Long filters require more computing time than short ones. Furthermore, the (dyadic) WT incorporates the aspect of iteration: the low-pass filter defines an approximation of the original signal which contains only half as many coefficients. This approximation successively builds the input for the next approximation. For compression purposes, coefficients in the time-scale domain are discarded and the synthesis quality improves with the number of iterations on the approximation. Finally, the WT is mathematically defined only within a signal; image applications thus need to solve the boundary problem. Depending on the selected boundary policy, the number of iterations in a WT might vary with the filter length. Moreover, the longer the filter length, the more important the boundary policy becomes.

In this work, we investigate the effects of three different boundary policies in combination with different wavelet filter banks on a number of gray-scale images. A first determining factor is the visual perception of a decoded image. As we will see, although the quality varies strongly with the selected image, for a given image it remains relatively unconcerned about the parameter settings. A second crucial factor is therefore the expected compression rate. Finally, the cost of implementation 
weights these two benchmarks. Our empirical evaluation leads us to recommend circular convolution as boundary treatment, contrary to JPEG-2000.

The article is organized as follows. In Section 2, we cite related work in wavelet filter evaluation. Section 3 reviews the wavelet transform and details the aspects that are important for our survey. In Section 4, we present the technical evaluation of the wavelet transform and detail our results. The article ends in Section 5 with an outlook on future work.

\section{RELATED WORK}

Villasenor's group researches wavelet filters for image compression. In [VBL95], the focus is on biorthogonal filters, and the evaluation is based on the information preserved in the reference signal, while [GFBV97] focuses on a mathematically optimal quantizer step size. In [AK99], the evaluation is based on lossless as well as on subjective lossy compression performance, complexity and memory usage. Interpretation of why the observations are made is nevertheless lacking. Strutz has thoroughly researched the dyadic WT in [Str97]: the design and construction of different wavelet filters is investigated, as are good Huffman and arithmetic encoding strategies. An investigation of boundary policies, however, is lacking. The new standard JPEG-2000 proposes mirror padding (or: periodic symmetric extension) as the image boundary treatment [SCE00] [ITU00].

\section{THE WAVELET TRANSFORM}

A wavelet is an (ideally) compact function, i.e., outside a certain interval it vanishes. Implementations are based on the fast wavelet transform, where a given wavelet (i.e., mother wavelet) is shifted and dilated so as to provide a base in the function space. In other words, a one-dimensional function is transformed into a two-dimensional space, where it is approximated by coefficients that depend on time (determined by the translation parameter) and on scale, i.e., frequency (determined by the dilation parameter). - By convention, the notion of time is used even for signals that depend on location rather than on time. Thus, a wavelet-transformed image is also said to be located in the time-scale domain. - The localization of a wavelet in time spread $\left(\sigma_{t}\right)$ and frequency spread $\left(\sigma_{\omega}\right)$ has the property $\sigma_{t} \sigma_{\omega}=$ const. However, the resolution in time and frequency depends on the frequency. This is the so-called zoom phenomenon of the WT: it offers high temporal localization for high frequencies while offering good frequency resolution for low frequencies. Consequently, the WT is especially well suited to analyze local variations such as those in still images: a high-frequency part of an image (e.g., a transition from bright foreground to black background) will be analyzed by short, high-amplitude wavelets. Low variations (e.g., gray value within the same object) will be analyzed by long, low-amplitude wavelets.

\subsection{Wavelet Transform and Filter Banks}

By introducing multiresolution, Mallat [Mal98] [Mal87] made an important contribution to the application of wavelet theory to multimedia: the transition from mathematical theory to filters. Multiresolution analysis is implemented via high-pass filters, resp. band-pass filters (i.e., wavelets) and low-pass filters (i.e., scaling functions). In this context, the wavelet transform of a signal can be realized with a filter bank via successive application of a 2-channel filter bank consisting of highpass and low-pass filters: the detail coefficients (resulting from the application of the high-pass, resp. band-pass filter) of every iteration step are kept apart, and the iteration starts again with the remaining approximation coefficients (from application of the low-pass filter) of the transform. This

multiresolution theory is 'per se' defined only for one-dimensional wavelets on one-dimensional signals. As still images are two-dimensional discrete signals and two-dimensional wavelet filter design 


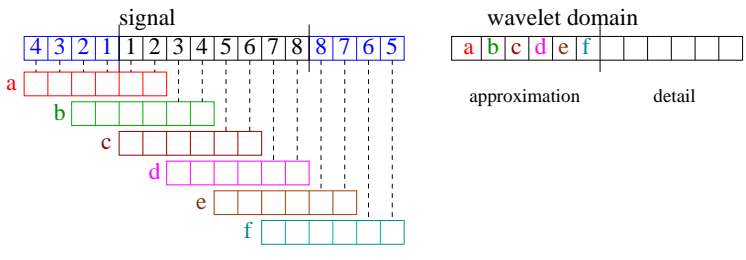

(a) Mirror Padding.

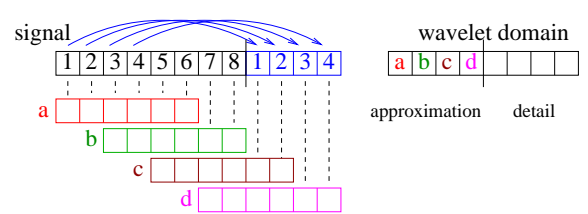

(b) Circular Convolution.

Figure 1: Mirror padding versus circular convolution for a signal of length of 8 and a filter with 6 taps. Here, the filter is a low-pass filter, thus the coefficients resulting from the convolution form the approximation entries. In (a), the padding results in inflated storage space in the wavelet domain. In (b), the approximation contains half as many entries as the original signal. Together with the details, the entries of the wavelet domain require the same storage space as the original signal.

remains an active field of research [KS00] [KV95] [TK93], current implementations are restricted to separable filters. The successive convolution of filter and signal in both dimensions opens two potential iterations: standard decomposition (i.e., all approximations, even in mixed terms, are iterated) and non-standard decomposition (i.e., only the purely low-pass filtered parts of every approximation enter the iteration). In this work, we concentrate on the non-standard decomposition.

\subsection{Image Boundary}

A digital filter is applied to a signal by convolution. Convolution, however, is defined only within a signal. In order to result in a reversible wavelet transform, each signal coefficient must enter into filter_length $/ 2$ calculations of convolution (here, the subsampling process by factor 2 is already incorporated). Consequently, every filter longer than two entries, i.e., every filter except Haar, requires a solution for the boundary. Furthermore, images are signals of a relatively short length (in rows and columns), thus the boundary treatment is even more important than e.g. in audio coding. Two common boundary policies are padding and circular convolution.

Padding Policies. With padding, the coefficients of the signal on either border are padded with filter_length-2 coefficients (see Figure 1 (a)). Consequently, each signal coefficient enters into filter_length/2 calculations of convolution, and the transform is reversible. Many padding policies exist: constant padding, where the signal's boundary coefficient is padded; mirror padding, where the signal is mirrored at the boundary; spline padding, where the border coefficients are extended by spline interpolation, etc. All padding policies have in common that each iteration step physically increases the storage space in the wavelet domain. In [Wic98], a theoretical solution for the required storage space (depending on the signal, the filter bank and the iteration level) is presented. Nevertheless, its implementation remains sophisticated.

Circular Convolution. The idea of circular convolution is to 'wrap' the end of a signal to its beginning or vice versa (see Figure 1 (b)). In so doing, circular convolution is the only boundary treatment to maintain the number of coefficients for a WT, thus simplifying storage management ${ }^{1}$. A minor drawback is that the time information contained in the time-scale domain of the wavelet-

\footnotetext{
${ }^{1}$ Storage space, however, expands indirectly: an image can be stored with integers, while the coefficients in the time-scale domain require floats.
} 
transformed coefficients 'blurs': the coefficients in the time-scale domain that are next to the right border (resp. left border) also affect signal coefficients that are located on the left (resp. right).

The selected boundary policy has an important impact on the iteration behavior of the wavelet transform: convolving the signal with a filter is only reasonable for a signal length greater than the filter length, and each iteration step reduces the size of the approximating signal by a factor of 2 . This does not affect the iteration behavior of padding policies. With circular convolution, however, the decomposition depth varies with the filter length: the longer the filter, the fewer decomposition iterations are possible. For example, for an image of $256 \times 256$ pixels, the Daubechies -2 filter bank with 4 taps allows a decomposition depth of 7 , while the Daubechies-20 filter bank with 40 taps has reached signal length after only 3 decomposition levels, see the table below.

\begin{tabular}{||l|c|c||}
\hline \hline Filter Bank & Taps & It. levels \\
\hline \hline Daub-2 & 4 & 7 \\
Daub-3 & 6 & 6 \\
Daub-4 & 8 & 6 \\
Daub-5 & 10 & 5 \\
Daub-10 & 20 & 4 \\
Daub-15 & 30 & 4 \\
Daub-20 & 40 & 3 \\
\hline \hline
\end{tabular}

Thus, the evaluation presented in Tables 1 to 4 is based on a decomposition depth of level 8 for the two padding policies, while the decomposition depth for circular convolution varies from 7 to 3 , according to the selected filter length.

\section{EMPIRICAL EVALUATION}

\subsection{Setup}

The goal of our empirical evaluation was to find the best parameter settings for the choice of the wavelet filter bank and for the image boundary policy to implement. The performance was evaluated according to the criteria:

1. visual quality,

2. compression rate,

3. complexity of implementation.

The quality was rated based on the peak signal-to-noise ratio (PSNR) ${ }^{2}$. The compression rate was simulated by a simple quantization threshold: the higher the threshold, the more coefficients in the time-scale domain are discarded, the higher is the compression rate. More precisely, the threshold was carried out only on the parts of the image that have been high-pass filtered (resp. band-pass filtered) at least once. In other words, the approximation of the image was excluded from the thresholding due to its importance for the image synthesis.

Our evaluation was set up on the six gray-scale images of size $256 \times 256$ pixels demonstrated in Figure 2. These test images have been chosen in order to comply with different features:

\footnotetext{
${ }^{2}$ When $\operatorname{org}(x, y)$ depicts the pixel value of the original image at position $(x, y)$, and $\operatorname{dec}(x, y)$ denotes the pixel value of the decoded image at position $(x, y)$, then

$\operatorname{PSNR}[\mathrm{dB}]=10 \cdot \log \left(\frac{\sum_{x y} 255^{2}}{\sum_{x y}(\operatorname{org}(x, y)-\operatorname{dec}(x, y))^{2}}\right)$.
} 
- contain many small details: Mandrill, Goldhill,

- contain large uniform areas: Brain, Lena, Camera, House,

- be relatively symmetric at the left-right and top-bottom boundaries: Mandrill, Brain,

- be very asymmetric with regard to these boundaries: Lena, Goldhill, House,

- have sharp transitions between regions: Brain, Lena, Camera, House,

- contain large areas of texture: Mandrill, Lena, Goldhill, House.

\subsection{Results}

\section{Image-Dependent Analysis.}

The detailed evaluation results for the six test images are presented in Tables 1 and 2. Some interesting observations made from these two tables and their explanations are as follows:

- For a given image and a given quantization threshold, the PSNR remains astonishingly constant for different filter banks and different boundary policies.

- At high thresholds, 'Mandrill' and 'Goldhill' yield the worst quality. This is due to the large amount of details in both images.

- 'House' produces the overall best quality at a given threshold. This is due to its large uniform areas.

- Due to their symmetry, 'Mandrill' and 'Brain' show good quality results with padding policies.

- The percentage of discarded information at a given threshold is far higher for 'Brain' than for 'Mandrill'. This is due to the black uniform background of 'Brain', which produces small coefficients in the time-scale domain compared to the many small details in 'Mandrill' which produce large coefficients and thus do not fall below the threshold.

- With regard to the heuristic for compression, and for a given image and boundary policy, Table 2 reveals that

- the compression ratio for zero padding increases with increasing filter length,

- the compression ratio for mirror padding decreases with increasing filter length,

- the compression ratio for circular convolution varies, but most often stays almost constant.

The explanation is as follows. Padding an image with zeros, i.e., black pixel values, is most often a sharp contrast to the original image, thus the sharp transition between the signal and the padding coefficients results in large coefficients in the fine scales, while the coarse scales remain unaffected. This observation, however, is put into a different perspective for longer filters: with longer filters, the constant run of zeros at the boundary does not show strong variations, and the detail coefficients in the time-scale domain thus remain small. Hence, a given threshold cuts off fewer coefficients when the filter is longer. With mirror padding, the padded coefficients for shorter filters represent a good heuristic for the signal near the boundary. Increasing filter length and accordingly longer padded areas, however, introduces too much 'false' detail information into the signal, resulting in many large detail coefficients that 'survive' the threshold.

\section{Image-Independent Analysis}

The above examples reveal that most phenomena are signal-dependent. As a signal-dependent determination of best-suited parameters remains academic, our further reflections are made on the average image quality and the average amount of discarded information as presented in Tables 3 and 4 and the corresponding Figures 3 and 4 . 
Figure 3 visualizes the coding quality of the images, averaged over the six test images. The four plots represent the quantization thresholds $10,20,45$ and 85 . In each graphic, the visual quality (quantified via PSNR) is plotted against the filter length of the Daubechies wavelet filters. The three boundary policies: zero padding, mirror padding and circular convolution are regarded separately. The plots obviously reveal that the quality decreases with an increasing threshold. More important are the following statements:

- Within a given threshold, and for a given boundary policy, the PSNR remains almost constant. This means that the quality of the coding process does not or hardly depends on the selected wavelet filter bank.

- Within a given threshold, mirror padding produces the best results, followed by circular convolution. Zero padding performs worst.

- The gap between the performance of the boundary policies increases with an increasing threshold.

Nevertheless, the differences observed above with $0.28 \mathrm{~dB}$ maximum gap (at threshold $=85$ and filter length $=40$ ) are so marginal that they do not actually influence the visual perception.

As the visual perception is neither influenced by the choice of filter nor by the boundary policy, the coding performance has been studied as a second benchmark. The following observations are made from Figure 4. With a short filter length (4 to 10 taps), the compression ratio is almost identical for the different boundary policies. This is not astonishing, as short filters involve only little boundary treatment, and the relative importance of the boundary coefficients with regard to the signal coefficients is negligible. More important for our investigation are:

- The compression heuristic for each of the three boundary policies is inversely proportional to their quality performance. In other words, mirror padding discards the least number of coefficients at a given quantization threshold, while zero padding discards the most.

- With an increasing threshold, the gap between the compression ratios of the three policies narrows.

In the overall evaluation, we have seen that mirror padding performs best with regard to quality, while it performs worst with regard to compression. Inversely, zero padding performs best with regard to compression and worst with regard to quality. Circular convolution holds the midway in both aspects. On the other hand, the gap in compression is by far superior to the differences in quality. If we now call to mind the coding complexity of the padding approaches, compared to the ease of implementation of circular convolution (cf. Section 3.2), we strongly recommend to implement circular convolution as the boundary policy in image coding.

\section{CONCLUSION}

We have discussed and evaluated the strengths and weaknesses of different boundary policies in relation to different orthogonal wavelet filter banks. In opposition to the JPEG-2000 coding standard, where mirror padding is suggested for boundary treatment, we have proven that circular convolution performance is superior in the overall combination of quality performance, compression performance and ease of implementation.

In future work, we will improve our heuristic on the compression rate and rely on the calculation of a signal's entropy as it is presented in [WM01] and [Str97]. 


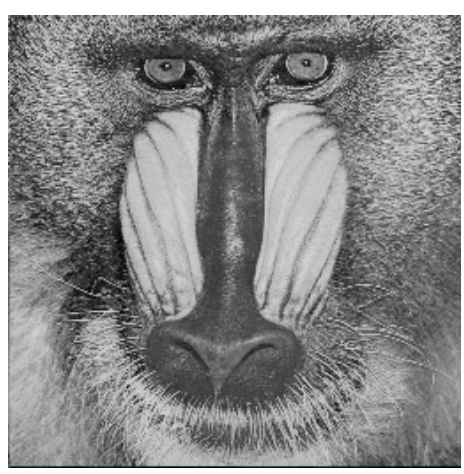

(a) Mandrill.

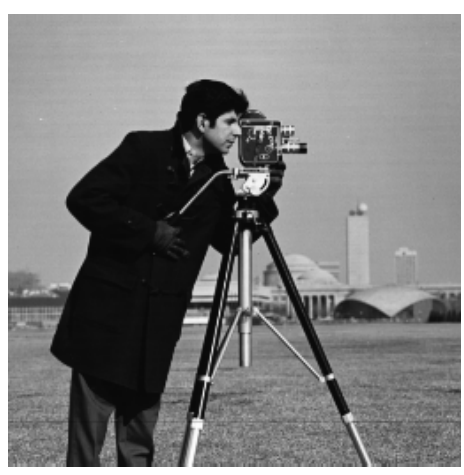

(d) Camera.

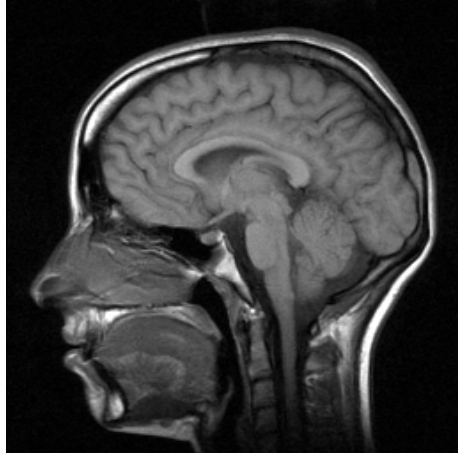

(b) Brain.

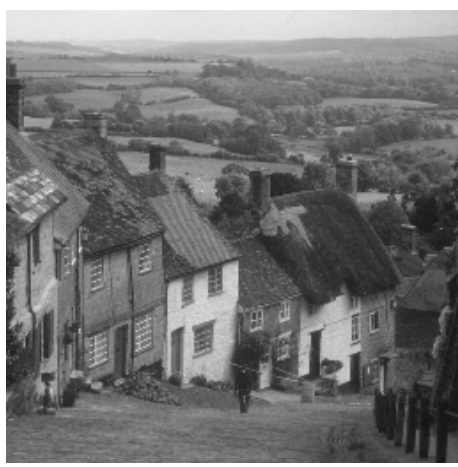

(e) Goldhill.

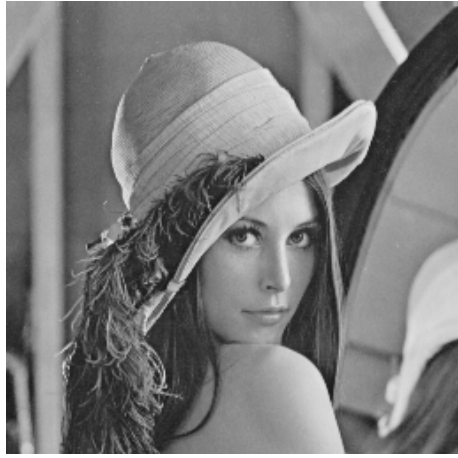

(c) Lena.

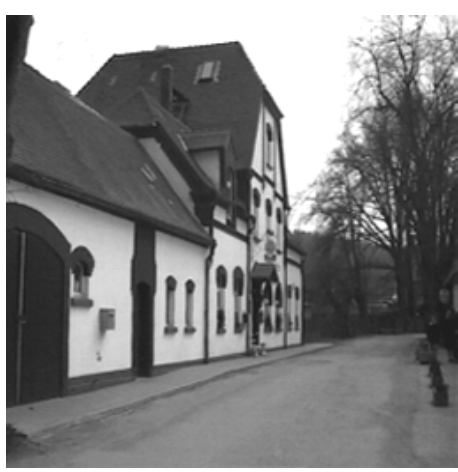

(f) House.

Figure 2: Test images. The test images (a) and (b) are relatively symmetric at their left-right and at their top-bottom boundary, while the images (c) - (f) are not. 


\begin{tabular}{|c|c|c|c|c|c|c|c|c|c|}
\hline & \multicolumn{9}{|c|}{ Quality of visual perception - PSNR [dB] } \\
\hline & \multicolumn{9}{|c|}{$\overline{\text { Image }}$} \\
\hline & \multicolumn{3}{|c|}{ Mandrill } & \multicolumn{3}{|c|}{ Brain } & \multicolumn{3}{|c|}{ Lena } \\
\hline \multirow[t]{2}{*}{ Wavelet } & $\begin{array}{c}\text { zero } \\
\text { padding }\end{array}$ & $\begin{array}{c}\text { mirror } \\
\text { padding }\end{array}$ & $\begin{array}{l}\text { circular } \\
\text { convol. }\end{array}$ & $\begin{array}{c}\text { zero } \\
\text { padding }\end{array}$ & $\begin{array}{c}\text { mirror } \\
\text { padding }\end{array}$ & $\begin{array}{l}\text { circular } \\
\text { convol. }\end{array}$ & $\begin{array}{c}\text { zero } \\
\text { padding }\end{array}$ & $\begin{array}{c}\text { mirror } \\
\text { padding }\end{array}$ & $\begin{array}{l}\text { circular } \\
\text { convol. }\end{array}$ \\
\hline & \multicolumn{9}{|c|}{ Threshold: $10-$ Excellent overall quality } \\
\hline Daub-2 & 18.012 & 17.996 & 18.238 & 18.141 & 18.151 & 18.197 & 16.392 & 16.288 & 16.380 \\
\hline Daub-3 & 18.157 & 18.187 & 18.221 & 18.429 & 18.434 & 18.433 & 16.391 & 16.402 & 16.350 \\
\hline Daub-4 & 18.169 & 18.208 & 17.963 & 18.353 & 18.340 & 18.248 & 16.294 & 16.355 & 16.260 \\
\hline Daub-5 & 18.173 & 18.167 & 18.186 & 18.279 & 18.280 & 18.259 & 16.543 & 16.561 & 16.527 \\
\hline Daub-10 & 17.977 & 17.959 & 18.009 & 18.291 & 18.300 & 18.479 & 16.249 & 16.278 & 16.214 \\
\hline Daub-15 & 17.938 & 17.934 & 18.022 & 18.553 & 18.543 & 18.523 & 16.267 & 16.304 & 16.288 \\
\hline \multirow[t]{2}{*}{ Daub-20 } & 17.721 & 17.831 & 18.026 & 18.375 & 18.357 & 18.466 & 16.252 & 16.470 & 16.238 \\
\hline & \multicolumn{9}{|c|}{ Threshold: $20-$ Good overall quality } \\
\hline Daub-2 & 14.298 & 14.350 & 14.403 & 16.610 & 16.611 & 16.577 & 14.775 & 14.765 & 14.730 \\
\hline Daub-3 & 14.414 & 14.469 & 14.424 & 16.743 & 16.755 & 16.721 & 14.758 & 14.817 & 14.687 \\
\hline Daub-4 & 14.231 & 14.239 & 14.276 & 16.637 & 16.628 & 16.734 & 14.862 & 14.918 & 14.735 \\
\hline Daub-5 & 14.257 & 14.216 & 14.269 & 16.747 & 16.751 & 16.854 & 14.739 & 14.946 & 14.815 \\
\hline Daub-10 & 14.268 & 14.274 & 14.360 & 16.801 & 16.803 & 16.878 & 14.624 & 14.840 & 14.699 \\
\hline Daub-15 & 14.246 & 14.258 & 14.300 & 16.822 & 16.810 & 16.852 & 14.395 & 14.631 & 14.477 \\
\hline Daub-20 & 14.046 & 14.065 & 14.227 & 16.953 & 16.980 & 16.769 & 14.252 & 14.597 & 14.353 \\
\hline & & & & shold: 45 & Mediun & verall qu & & & \\
\hline Daub-2 & 10.905 & 10.885 & 10.910 & 14.815 & 14.816 & 14.747 & 13.010 & 13.052 & 12.832 \\
\hline Daub-3 & 10.988 & 10.970 & 10.948 & 15.187 & 15.150 & 15.052 & 12.766 & 13.138 & 12.903 \\
\hline Daub-4 & 10.845 & 10.839 & 10.885 & 15.014 & 15.029 & 15.056 & 12.820 & 13.132 & 12.818 \\
\hline Daub-5 & 10.918 & 10.969 & 10.949 & 15.036 & 15.031 & 14.999 & 12.913 & 13.301 & 12.983 \\
\hline Daub-10 & 10.907 & 10.929 & 10.913 & 14.989 & 15.013 & 15.212 & 12.447 & 13.066 & 12.795 \\
\hline Daub-15 & 10.845 & 10.819 & 10.815 & 15.093 & 15.133 & 15.064 & 12.577 & 12.954 & 12.686 \\
\hline Daub-20 & 10.784 & 10.872 & 10.843 & 14.975 & 14.934 & 14.882 & 12.299 & 12.877 & 12.640 \\
\hline & & & & ceshold: & $-\mathrm{Bad}$ & rall qual & & & \\
\hline Daub-2 & 9.095 & 9.121 & 9.135 & 13.615 & 13.621 & 13.783 & 11.587 & 11.902 & 11.577 \\
\hline Daub-3 & 9.206 & 9.184 & 9.124 & 13.787 & 13.784 & 13.759 & 11.437 & 11.793 & 11.516 \\
\hline Daub-4 & 9.160 & 9.152 & 9.168 & 13.792 & 13.815 & 13.808 & 11.539 & 11.806 & 11.636 \\
\hline Daub-5 & 9.171 & 9.208 & 9.203 & 13.837 & 13.850 & 13.705 & 11.692 & 11.790 & 11.872 \\
\hline Daub-10 & 9.207 & 9.193 & 9.206 & 13.870 & 13.922 & 14.042 & 11.128 & 11.430 & 11.555 \\
\hline Daub-15 & 9.083 & 9.161 & 9.126 & 13.731 & 13.795 & 13.917 & 11.128 & 11.610 & 11.475 \\
\hline Daub-20 & 9.071 & 9.142 & 9.204 & 13.852 & 13.800 & 13.974 & 11.142 & 11.694 & 11.597 \\
\hline & & & & & Image & & & & \\
\hline & & Camera & & & Goldhill & & & House & \\
\hline Wavelet & $\begin{array}{c}\text { zero } \\
\text { padding } \\
\end{array}$ & $\begin{array}{c}\text { mirror } \\
\text { padding }\end{array}$ & $\begin{array}{l}\text { circular } \\
\text { convol. }\end{array}$ & $\begin{array}{c}\text { zero } \\
\text { padding } \\
\end{array}$ & $\begin{array}{c}\text { mirror } \\
\text { padding }\end{array}$ & $\begin{array}{l}\text { circular } \\
\text { convol. }\end{array}$ & $\begin{array}{c}\text { zero } \\
\text { padding }\end{array}$ & $\begin{array}{c}\text { mirror } \\
\text { padding }\end{array}$ & $\begin{array}{l}\text { circular } \\
\text { convol. }\end{array}$ \\
\hline & & & & shold: 10 & Excellen & overall qu & ity & & \\
\hline Daub-2 & 17.334 & 17.346 & 17.371 & 16.324 & 16.266 & 16.412 & 19.575 & 19.563 & 19.608 \\
\hline Daub-3 & 17.532 & 17.560 & 17.625 & 16.322 & 16.296 & 16.358 & 19.640 & 19.630 & 19.621 \\
\hline Daub-4 & 17.529 & 17.591 & 17.577 & 16.241 & 16.212 & 16.342 & 19.560 & 19.558 & 19.584 \\
\hline Daub-5 & 17.489 & 17.448 & 17.389 & 16.214 & 16.193 & 16.154 & 19.613 & 19.555 & 19.566 \\
\hline Daub-10 & 17.539 & 17.541 & 17.383 & 16.307 & 16.223 & 16.317 & 19.482 & 19.388 & 19.732 \\
\hline Daub-15 & 17.747 & 17.530 & 17.523 & 16.012 & 16.067 & 16.033 & 19.653 & 19.671 & 19.726 \\
\hline Daub-20 & 17.474 & 17.527 & 17.484 & 16.322 & 16.245 & 16.319 & 19.550 & 19.495 & 19.524 \\
\hline & & & & reshold: 2 & - Good & erall qua & & & \\
\hline Daub-2 & 14.387 & 14.365 & 14.396 & 13.937 & 13.940 & 13.898 & 17.446 & 17.480 & 17.471 \\
\hline Daub-3 & 14.473 & 14.452 & 14.426 & 13.872 & 13.892 & 13.858 & 17.525 & 17.594 & 17.612 \\
\hline Daub-4 & 14.438 & 14.438 & 14.430 & 13.828 & 13.836 & 13.753 & 17.468 & 17.647 & 17.351 \\
\hline Daub-5 & 14.460 & 14.505 & 14.427 & 13.743 & 13.743 & 13.711 & 17.454 & 17.458 & 17.465 \\
\hline Daub-10 & 14.468 & 14.400 & 14.409 & 13.762 & 13.785 & 13.798 & 17.592 & 17.635 & 17.689 \\
\hline Daub-15 & 14.408 & 14.406 & 14.414 & 13.687 & 13.730 & 13.697 & 17.260 & 17.276 & 17.266 \\
\hline Daub-20 & 14.384 & 14.370 & 14.362 & 13.700 & 13.782 & 13.731 & 17.476 & 17.449 & 17.240 \\
\hline & & & & hold: 45 & Mediun & verall qu & & & \\
\hline Daub-2 & 12.213 & 12.242 & 12.131 & 12.033 & 12.034 & 11.876 & 15.365 & 15.437 & 15.155 \\
\hline Daub-3 & 12.032 & 12.122 & 12.188 & 11.961 & 12.006 & 11.889 & 14.957 & 15.476 & 15.118 \\
\hline Daub-4 & 12.150 & 12.178 & 12.145 & 11.855 & 11.891 & 11.925 & 14.906 & 15.080 & 15.180 \\
\hline Daub-5 & 12.077 & 12.133 & 12.120 & 11.848 & 11.844 & 11.801 & 15.159 & 15.382 & 15.244 \\
\hline Daub-10 & 12.061 & 12.197 & 12.093 & 11.760 & 11.917 & 11.726 & 14.776 & 15.246 & 14.872 \\
\hline Daub-15 & 12.074 & 12.059 & 12.176 & 11.725 & 11.855 & 11.753 & 14.810 & 15.090 & 14.969 \\
\hline Daub-20 & 11.798 & 11.975 & 12.048 & 11.763 & 11.803 & 11.703 & 14.420 & 15.033 & 14.609 \\
\hline & & & & eshold: & $-\overline{B a d} 0$ & rall qual & & & \\
\hline Daub-2 & 11.035 & 11.161 & 11.041 & 10.791 & 10.805 & 10.844 & 13.530 & 13.804 & 13.703 \\
\hline Daub-3 & 11.092 & 11.176 & 11.080 & 10.943 & 10.916 & 10.754 & 13.488 & 13.726 & 13.627 \\
\hline Daub-4 & 10.943 & 11.152 & 11.046 & 10.861 & 10.904 & 10.740 & 13.524 & 13.613 & 13.510 \\
\hline Daub-5 & 11.018 & 11.148 & 11.129 & 10.826 & 10.935 & 10.738 & 13.114 & 13.903 & 13.111 \\
\hline Daub-10 & 10.815 & 11.064 & 10.987 & 10.824 & 10.972 & 10.771 & 13.158 & 13.695 & 13.434 \\
\hline Daub-15 & 10.779 & 11.005 & 10.982 & 10.737 & 10.838 & 10.607 & 13.073 & 13.357 & 13.123 \\
\hline Daub-20 & 10.688 & 11.031 & 11.090 & 10.709 & 10.819 & 10.766 & 13.173 & 13.257 & 13.678 \\
\hline
\end{tabular}

Table 1: Detailed results of the quality evaluation with the PSNR on the six test images. The mean values over the images for a fixed wavelet filter bank and a fixed boundary policy are given in Table 3. 


\begin{tabular}{|c|c|c|c|c|c|c|c|c|c|}
\hline & \multicolumn{9}{|c|}{ Discarded information in the time-scale domain due to the threshold - Percentage [\%] } \\
\hline & \multicolumn{9}{|c|}{ Image } \\
\hline & \multicolumn{3}{|c|}{ Mandrill } & \multicolumn{3}{|c|}{ Brain } & \multicolumn{3}{|c|}{ Lena } \\
\hline \multirow[t]{2}{*}{ Wavelet } & $\begin{array}{c}\text { zero } \\
\text { padding } \\
\end{array}$ & $\begin{array}{c}\text { mirror } \\
\text { padding }\end{array}$ & $\begin{array}{l}\text { circular } \\
\text { convol. }\end{array}$ & $\begin{array}{c}\text { zero } \\
\text { padding }\end{array}$ & $\begin{array}{c}\text { mirror } \\
\text { padding }\end{array}$ & $\begin{array}{l}\text { circular } \\
\text { convol. }\end{array}$ & $\begin{array}{c}\text { zero } \\
\text { padding }\end{array}$ & $\begin{array}{c}\text { mirror } \\
\text { padding }\end{array}$ & $\begin{array}{l}\text { circular } \\
\text { convol. }\end{array}$ \\
\hline & \multicolumn{9}{|c|}{ Threshold: $10-$ Excellent overall quality } \\
\hline Daub-2 & 42 & 41 & $\overline{41}$ & 83 & 83 & 83 & 78 & 79 & 79 \\
\hline Daub-3 & 43 & 42 & 42 & 84 & 84 & 84 & 78 & 80 & 80 \\
\hline Daub-4 & 44 & 42 & 41 & 85 & 84 & 84 & 78 & 79 & 79 \\
\hline Daub-5 & 45 & 41 & 41 & 85 & 84 & 84 & 79 & 79 & 80 \\
\hline Daub-10 & 53 & 38 & 41 & 87 & 82 & 84 & 79 & 74 & 78 \\
\hline Daub-15 & 59 & 35 & 40 & 88 & 78 & 82 & 82 & 69 & 77 \\
\hline \multirow[t]{2}{*}{ Daub-20 } & 65 & 32 & 40 & 89 & 74 & 83 & 83 & 64 & 77 \\
\hline & \multicolumn{9}{|c|}{ Threshold: $20-$ Good overall quality } \\
\hline Daub-2 & 63 & 63 & 63 & 91 & 91 & 91 & 87 & 89 & 88 \\
\hline Daub-3 & 64 & 63 & 64 & 92 & 91 & 91 & 87 & 89 & 89 \\
\hline Daub-4 & 65 & 63 & 63 & 92 & 91 & 91 & 87 & 88 & 89 \\
\hline Daub-5 & 66 & 62 & 63 & 92 & 91 & 91 & 87 & 90 & 89 \\
\hline Daub-10 & 70 & 58 & 63 & 93 & 89 & 91 & 88 & 83 & 88 \\
\hline Daub-15 & 74 & 56 & 62 & 93 & 86 & 91 & 89 & 79 & 88 \\
\hline Daub-20 & 78 & 51 & 63 & 94 & 82 & 91 & 90 & 74 & 88 \\
\hline & & & & shold: 45 & - Medium & verall qu & & & \\
\hline Daub-2 & 86 & 86 & 87 & 96 & 96 & 96 & 94 & 95 & 95 \\
\hline Daub-3 & 86 & 86 & 87 & 96 & 96 & 96 & 94 & 95 & 95 \\
\hline Daub-4 & 87 & 86 & 87 & 96 & 96 & 96 & 94 & 95 & 96 \\
\hline Daub-5 & 87 & 85 & 87 & 96 & 96 & 96 & 95 & 94 & 96 \\
\hline Daub-10 & 88 & 82 & 87 & 97 & 94 & 96 & 94 & 91 & 96 \\
\hline Daub-15 & 90 & 79 & 87 & 97 & 91 & 96 & 95 & 88 & 96 \\
\hline Daub-20 & 92 & 74 & 87 & 97 & 89 & 96 & 96 & 83 & 96 \\
\hline & & & & reshold: & - Bad 0 & rall qual & & & \\
\hline Daub-2 & $\overline{96}$ & $\overline{96}$ & $\overline{97}$ & 98 & 98 & 98 & $\overline{97}$ & 98 & $\overline{98}$ \\
\hline Daub-3 & 96 & 96 & 97 & 98 & 98 & 98 & 97 & 98 & 98 \\
\hline Daub-4 & 96 & 96 & 97 & 98 & 98 & 98 & 97 & 97 & 98 \\
\hline Daub-5 & 96 & 95 & 97 & 98 & 98 & 98 & 98 & 97 & 98 \\
\hline Daub-10 & 97 & 93 & 97 & 98 & 97 & 98 & 97 & 94 & 98 \\
\hline Daub-15 & 97 & 91 & 97 & 98 & 95 & 98 & 98 & 92 & 98 \\
\hline Daub-20 & 97 & 86 & 98 & 98 & 93 & 99 & 98 & 88 & 99 \\
\hline & & & & & Image & & & & \\
\hline & & Camera & & & Goldhill & & & House & \\
\hline Wavelet & $\begin{array}{c}\text { zero } \\
\text { padding }\end{array}$ & $\begin{array}{c}\text { mirror } \\
\text { padding }\end{array}$ & $\begin{array}{l}\text { circular } \\
\text { convol. }\end{array}$ & $\begin{array}{c}\text { zero } \\
\text { padding }\end{array}$ & $\begin{array}{c}\text { mirror } \\
\text { padding }\end{array}$ & $\begin{array}{l}\text { circular } \\
\text { convol. }\end{array}$ & $\begin{array}{c}\text { zero } \\
\text { padding }\end{array}$ & $\begin{array}{c}\text { mirror } \\
\text { padding }\end{array}$ & $\begin{array}{l}\text { circular } \\
\text { convol. }\end{array}$ \\
\hline & & & & shold: 10 & - Excellen & overall qu & ity & & \\
\hline Daub-2 & 78 & 80 & 79 & 70 & 71 & 70 & 79 & 80 & 80 \\
\hline Daub-3 & 77 & 79 & 78 & 70 & 71 & 71 & 79 & 80 & 80 \\
\hline Daub-4 & 77 & 79 & 78 & 71 & 71 & 70 & 79 & 80 & 79 \\
\hline Daub-5 & 77 & 78 & 78 & 71 & 71 & 70 & 79 & 79 & 79 \\
\hline Daub-10 & 77 & 74 & 76 & 73 & 67 & 69 & 80 & 72 & 78 \\
\hline Daub-15 & 80 & 71 & 75 & 77 & 63 & 68 & 82 & 66 & 77 \\
\hline Daub-20 & 81 & 66 & 74 & 79 & 58 & 68 & 83 & 59 & 76 \\
\hline & & & & reshold: 2 & - Good & erall qua & & & \\
\hline Daub-2 & 86 & 88 & 88 & 85 & 87 & 86 & 87 & 88 & 88 \\
\hline Daub-3 & 86 & 88 & 88 & 85 & 87 & 86 & 87 & 88 & 88 \\
\hline Daub-4 & 86 & 88 & 88 & 86 & 86 & 86 & 87 & 88 & 87 \\
\hline Daub-5 & 86 & 87 & 88 & 86 & 86 & 86 & 87 & 87 & 88 \\
\hline Daub-10 & 86 & 85 & 87 & 86 & 83 & 86 & 87 & 81 & 87 \\
\hline Daub-15 & 88 & 82 & 86 & 89 & 79 & 86 & 89 & 75 & 87 \\
\hline Daub-20 & 88 & 78 & 86 & 89 & 73 & 86 & 89 & 69 & 87 \\
\hline & & & & shold: 45 & - Medium & verall qu & & & \\
\hline Daub-2 & $\overline{93}$ & 95 & 95 & 94 & 96 & 95 & $\overline{93}$ & 95 & 94 \\
\hline Daub-3 & 93 & 95 & 95 & 95 & 96 & 95 & 94 & 95 & 95 \\
\hline Daub-4 & 94 & 95 & 95 & 95 & 95 & 95 & 94 & 94 & 95 \\
\hline Daub-5 & 94 & 94 & 95 & 95 & 95 & 96 & 94 & 94 & 95 \\
\hline Daub-10 & 93 & 93 & 95 & 95 & 92 & 96 & 94 & 89 & 95 \\
\hline Daub-15 & 94 & 91 & 95 & 95 & 89 & 96 & 95 & 84 & 94 \\
\hline Daub-20 & 95 & 88 & 95 & 96 & 85 & 96 & 95 & 78 & 95 \\
\hline & & & & reshold: & $5-\mathrm{Bad} 0$ & rall qual & & & \\
\hline Daub-2 & 97 & 98 & 98 & 97 & 98 & 98 & 97 & 98 & $\overline{98}$ \\
\hline Daub-3 & 97 & 98 & 98 & 98 & 98 & 98 & 97 & 97 & 97 \\
\hline Daub-4 & 97 & 98 & 98 & 98 & 98 & 98 & 97 & 97 & 98 \\
\hline Daub-5 & 97 & 97 & 98 & 98 & 98 & 99 & 97 & 97 & 98 \\
\hline Daub-10 & 97 & 96 & 98 & 98 & 96 & 99 & 97 & 93 & 98 \\
\hline Daub-15 & 97 & 95 & 98 & 98 & 93 & 99 & 97 & 89 & 98 \\
\hline Daub-20 & 98 & 93 & 98 & 98 & 90 & 99 & 98 & 84 & 99 \\
\hline
\end{tabular}

Table 2: Heuristic for the compression rate of the coding parameters of Table 1: the higher the percentage of discarded information in the time-scale domain is, the higher is the compression ratio. The mean values over the images for a fixed wavelet filter bank and a fixed boundary policy are given in Table 4. 


\begin{tabular}{|c|c|c|c|c|c|c|}
\hline & \multicolumn{6}{|c|}{ Average image quality - PSNR $[\mathrm{dB}]$} \\
\hline Wavelet & $\begin{array}{c}\text { zero } \\
\text { padding }\end{array}$ & $\begin{array}{c}\text { mirror } \\
\text { padding }\end{array}$ & $\begin{array}{l}\text { circular } \\
\text { convol. }\end{array}$ & $\begin{array}{c}\text { zero } \\
\text { padding }\end{array}$ & $\begin{array}{c}\text { mirror } \\
\text { padding }\end{array}$ & $\begin{array}{l}\text { circular } \\
\text { convol. }\end{array}$ \\
\hline & \multicolumn{3}{|c|}{ Threshold 10} & \multicolumn{3}{|c|}{ Threshold 20} \\
\hline Daub-2 & 17.630 & 17.602 & 17.701 & 15.242 & 15.252 & 15.246 \\
\hline Daub-3 & 17.745 & 17.752 & 17.768 & 15.298 & 15.330 & 15.288 \\
\hline Daub-4 & 17.691 & 17.711 & 17.662 & 15.244 & 15.284 & 15.213 \\
\hline Daub-5 & 17.719 & 17.701 & 17.680 & 15.233 & 15.270 & 15.257 \\
\hline Daub-10 & 17.641 & 17.615 & 17.689 & 15.253 & 15.290 & 15.306 \\
\hline Daub-15 & 17.695 & 17.675 & 17.686 & 15.136 & 15.185 & 15.168 \\
\hline Daub-20 & 17.616 & 17.654 & 17.676 & 15.135 & 15.207 & 15.114 \\
\hline & \multicolumn{3}{|c|}{ Threshold 45} & \multicolumn{3}{|c|}{ Threshold 85} \\
\hline Daub-2 & 13.057 & 13.078 & 12.942 & 11.609 & 11.736 & 11.681 \\
\hline Daub-3 & 12.982 & 13.144 & 13.016 & 11.659 & 11.763 & 11.643 \\
\hline Daub-4 & 12.932 & 13.025 & 13.002 & 11.637 & 11.740 & 11.651 \\
\hline Daub-5 & 12.992 & 13.110 & 13.016 & 11.610 & 11.806 & 11.626 \\
\hline Daub-10 & 12.823 & 13.061 & 12.935 & 11.500 & 11.713 & 11.666 \\
\hline Daub-15 & 12.854 & 12.985 & 12.911 & 11.422 & 11.628 & 11.538 \\
\hline Daub-20 & 12.673 & 12.916 & 12.788 & 11.439 & 11.624 & 11.718 \\
\hline
\end{tabular}

Table 3: Average quality of the six test images. Figure 3 gives a more 'readable' plot of these digits.
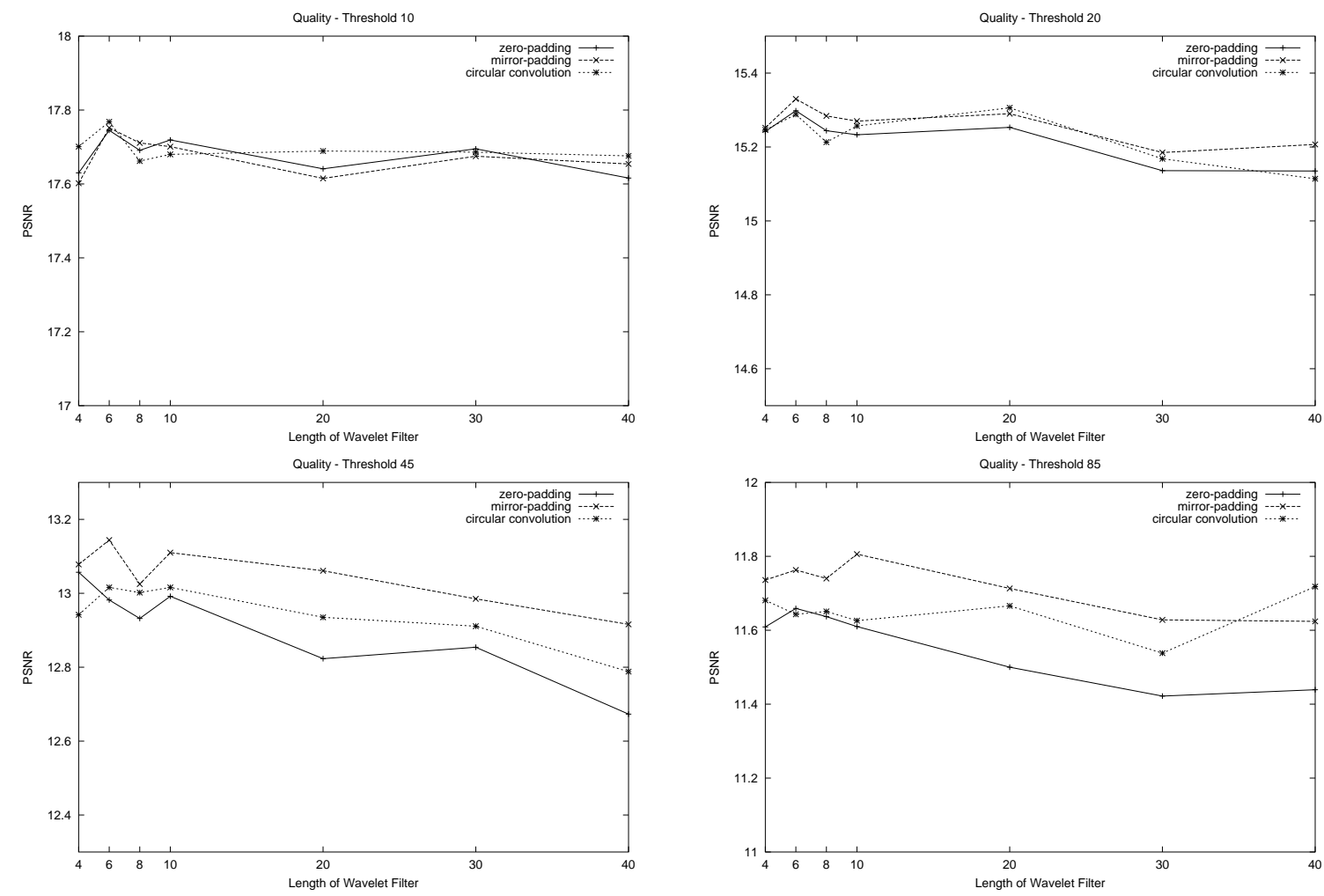

Figure 3: Visual quality of the test images at the quantization thresholds $10,20,45$ and 85 . The values are averaged over the six test images and correspond to Table 3. 


\begin{tabular}{|c|c|c|c|c|c|c|}
\hline & \multicolumn{6}{|c|}{ Average discarded information - Percentage [\%] } \\
\hline Wavelet & $\begin{array}{c}\text { zero } \\
\text { padding }\end{array}$ & $\begin{array}{c}\text { mirror } \\
\text { padding }\end{array}$ & $\begin{array}{l}\text { circular } \\
\text { convol. }\end{array}$ & $\begin{array}{c}\text { zero } \\
\text { padding }\end{array}$ & $\begin{array}{c}\text { mirror } \\
\text { padding }\end{array}$ & $\begin{array}{l}\text { circular } \\
\text { convol. }\end{array}$ \\
\hline & \multicolumn{3}{|c|}{ Threshold 10} & \multicolumn{3}{|c|}{ Threshold 20} \\
\hline Daub-2 & 72.0 & 72.3 & 72.0 & 83.2 & 84.3 & 84.0 \\
\hline Daub-3 & 71.8 & 72.7 & 72.5 & 83.5 & 84.3 & 84.3 \\
\hline Daub-4 & 72.3 & 72.5 & 71.8 & 83.8 & 84.0 & 84.0 \\
\hline Daub-5 & 72.7 & 72.0 & 72.0 & 84.0 & 83.8 & 84.2 \\
\hline Daub-10 & 74.8 & 67.8 & 71.0 & 85.0 & 79.8 & 83.7 \\
\hline Daub-15 & 78.0 & 63.7 & 69.8 & 87.0 & 76.2 & 83.3 \\
\hline Daub-20 & 80.0 & 58.8 & 69.7 & 88.0 & 71.2 & 83.5 \\
\hline & \multicolumn{3}{|c|}{ Threshold 45} & \multicolumn{3}{|c|}{ Threshold 85} \\
\hline Daub-2 & 92.7 & 93.8 & 93.7 & 97.0 & 97.7 & 97.8 \\
\hline Daub-3 & 93.0 & 93.8 & 93.8 & 97.2 & 97.5 & 97.7 \\
\hline Daub-4 & 93.3 & 93.5 & 94.0 & 97.2 & 97.3 & 97.8 \\
\hline Daub-5 & 93.5 & 93.0 & 94.2 & 97.3 & 97.0 & 98.0 \\
\hline Daub-10 & 93.5 & 90.2 & 94.2 & 97.3 & 94.8 & 98.0 \\
\hline Daub-15 & 94.3 & 87.0 & 94.0 & 97.5 & 92.5 & 98.0 \\
\hline Daub-20 & 95.2 & 82.8 & 94.2 & 97.8 & 89.0 & 98.7 \\
\hline
\end{tabular}

Table 4: Average bitrate heuristic of the six test images. Figure 4 gives a more 'readable' plot of these digits.
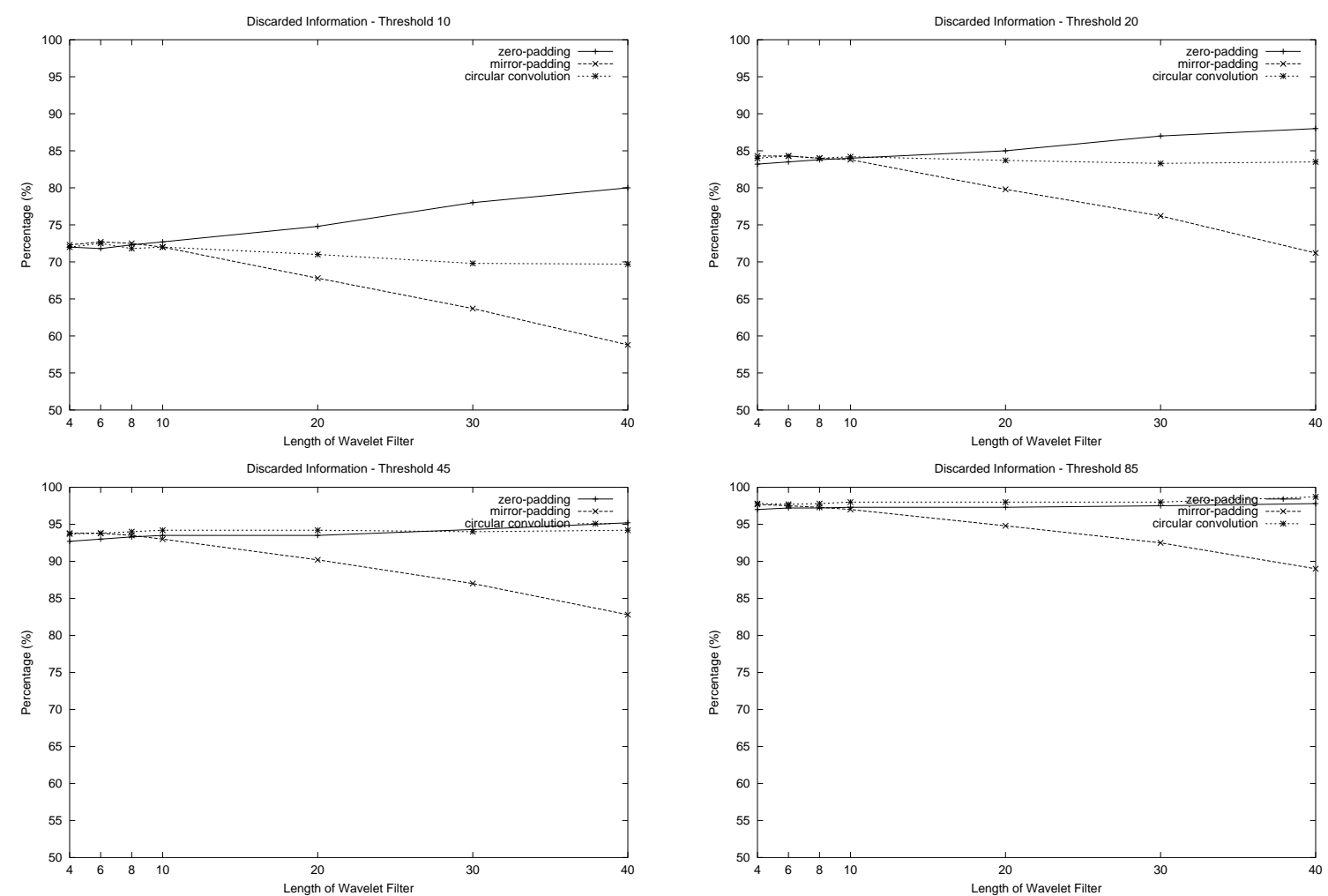

Figure 4: Average bitrate heuristic of the test images at the quantization thresholds 10, 20, 45 and 85. The values are averaged over the six test images and correspond to Table 4. 


\section{References}

[AK99] Michael D. Adams and Faouzi Kossentini. Performance Evaluation of Reversible Integerto-Integer Wavelet Transforms for Image Compression. In Proc. IEEE Data Compression Conference, page $514 \mathrm{ff}$, Snowbird, Utah, March 1999.

[Dau92] Ingrid Daubechies. Ten Lectures on Wavelets, volume 61. SIAM. Society for Industrial and Applied Mathematics, Philadelphia, Pennsylvania, 1992.

[GFBV97] Javier Garcia-Frias, Dan Benyamin, and John D. Villasenor. Rate Distortion Optimal Parameter Choice in a Wavelet Image Communication System. In Proc. International Conference on Image Processing (ICIP), pages 25-28, Santa Barbara, CA, October 1997.

[ITU00] ITU. JPEG 2000 Image Coding System. International Telecommunication Union, Final Committee Draft Version 1.0 - FCD15444-1 edition, March 2000.

[KS00] Jelena Kovačević and Wim Sweldens. Wavelet Families of Increasing Order in Arbitrary Dimensions. IEEE Transactions on Image Processing, 9(3):480-496, March 2000.

[KV95] Jelena Kovačević and Martin Vetterli. Nonseparable Two- and Three-Dimensional Wavelets. IEEE Transactions on Signal Processing, 43(5):1269-1273, May 1995.

[Mal87] Stéphane Mallat. A Compact Multiresolution Representation: The Wavelet Model. IEEE Computer Society Workshop on Computer Vision (WCV), 87:2-7, 1987.

[Mal98] Stéphane Mallat. A Wavelet Tour of Signal Processing. Academic Press, San Diego, CA, USA, 1998.

[SCE00] Athanassios N. Skodras, Charilaos A. Christopoulos, and Touradj Ebrahimi. JPEG2000: The Upcoming Still Image Compression Standard. In 11th Portuguese Conference on Pattern Recognition (RECPA00D), pages 359-366, Porto, Portugal, May 2000.

[Str97] Tilo Strutz. Untersuchungen zur skalierbaren Kompression von Bildsequenzen bei niedrigen Bitraten unter Verwendung der dyadischen Wavelet-Transformation. PhD thesis, Universität Rostock, Germany, May 1997.

[TK93] David B. H. Tay and N. G. Kingsbury. Flexible Design of Multidimensional Perfect Reconstruction FIR 2-Band Filters using Transformations of Variables. IEEE Transactions on Image Processing, 2(4):466-480, October 1993.

[VBL95] John D. Villasenor, Benjamin Belzer, and Judy Liao. Wavelet Filter Evaluation for Image Compression. IEEE Transactions on Image Processing, 2:1053-1060, August 1995.

[Wic98] Mladen Victor Wickerhauser. Adapted Wavelet Analysis from Theory to Software. A K Peters Ltd., Wellesley, Massachusetts, USA, 1998.

[WM01] Mathias Wien and Claudia Meyer. Adaptive Block Transform for Hybrid Video Coding. In Proc. SPIE Visual Communications and Image Processing, pages 153-162, San Jose, CA, January 2001. 